\title{
GALAXY COUNT MODELS AND THE EXTRAGALACTIC BACKGROUND LIGHT
}

\author{
T. Shanks \\ Physics Department \\ University of Durham \\ South Road \\ Durham DH1 3LE, UK
}

\begin{abstract}
In this review we first discuss the various ingredients of the non-evolving models for galaxy count observations. These ingredients include the K-corrections, the galaxy luminosity function and its dependence on galaxy colour, the local space density of galaxies and the cosmological deceleration parameter. Comparing the updated count model with the most recent galaxy count observations, the conclusion is still that at $B>22^{m}$ the $B$ (and the $R$ ) counts require very significant amounts of evolution to fit the observed, steep $\mathrm{n}(\mathrm{m})$ relations. We review models of galaxy luminosity evolution and also discuss the current debate as to whether $B \approx 23^{m}$ galaxies have $z \approx 1$ or $z \approx 3$.

We also consider recent suggestions that the form of Tyson's counts at $B=27^{m}$ may constrain the cosmological deceleration parameter; contrary to the suggestion of Koo (1989), it is proposed here that if the turnover seen in Tyson's B counts is real then this argues for high values of $\mathrm{q}_{0}$ and/or low galaxy formation redshifts. Finally, we consider the implications of these results for observations of the extragalactic background light (EBL) and conclude that in the ultraviolet (UV) and in the optical the contribution of faint galaxies to the EBL is likely to be small if the turnover in Tyson's counts is real. The galaxy count evidence gives less clear indications about the contribution of galaxies to the infrared EBL.
\end{abstract}

\section{INTRODUCTION}

If a galaxy number-count to a flux limit, $S_{\text {lim }}$ goes as a power law $S_{\text {lim }}{ }^{-\alpha}$ then the surface brightness associated with these galaxies goes as $S_{1 \mathrm{~lm}}{ }^{1-\alpha}$. In a Euclidean Universe this surface brightness diverges since $\alpha=1.5$ and the surface brightness goes as $S_{\text {lim }}{ }^{-0.5}$, leading to Olbers's Paradox. However, as is well known, the power-law slope $\alpha$ may be flattened by evolution as well as cosmology. Here we consider what recent models for galaxy number counts might mean for the presence of a detectable extragalactic background light due to galaxies.

Previously, simple models suggested that galaxies might provide a detectable EBL in the optical and in the UV. Paresce and Jakobsen (1980) showed that a model where every galaxy was assumed to be as blue as an Scd could give an integrated light close to the upper limits for the EBL in the optical and in the UV. They also suggested that QSOs might give, to order of magnitude, a detectable EBL. However, recently it has been found that the QSO B number count flattens at $B=19 .^{m} 5$ (e.g., Boyle et al., 1988), suggesting that at least in the optical bands the QSO EBL will not be detectable.

For galaxies, Cowie (1988) has shown that the EBL due to the integrated light of the $22^{m} \leq B \leq 27^{m}$ galaxies detected by Tyson (1988), amounts to $10 \%$ of the EBL upper limits in the optical (e.g., Dube et al., 1977). The aim of this paper is to give at least some qualitative idea of how much the rest of the upper limit at these and other wavelengths might be due to the contribution of galaxies. 
Therefore in Section 2 we first discuss the input parameters of the non-evolving models; these include the $\mathrm{K}$-corrections, the galaxy luminosity function, its normalization and the underlying cosmological model. In Section 3 we look at evolutionary models and in Section 4 we consider the debate over the possible redshift of the $B=24^{m}$ galaxies. In Sections 5 and 6 we conclude by discussing the implications of the galaxy count model results for cosmological models and for the faint galaxy contribution to the extragalactic background light.

\section{NUMBER COUNT MODEL PARAMETERS}

\subsection{K-corrections}

The first ingredient of a non-evolving model is the $\mathrm{K}$-correction; this correction accounts for the fact that at high redshift a different part of a galaxy's spectrum will be observed in the rest passband than for a zero redshift galaxy. The B-band K-corrections (and to a lesser extent the R) therefore depend on measurements of the spectral energy distribution of galaxies in the UV.

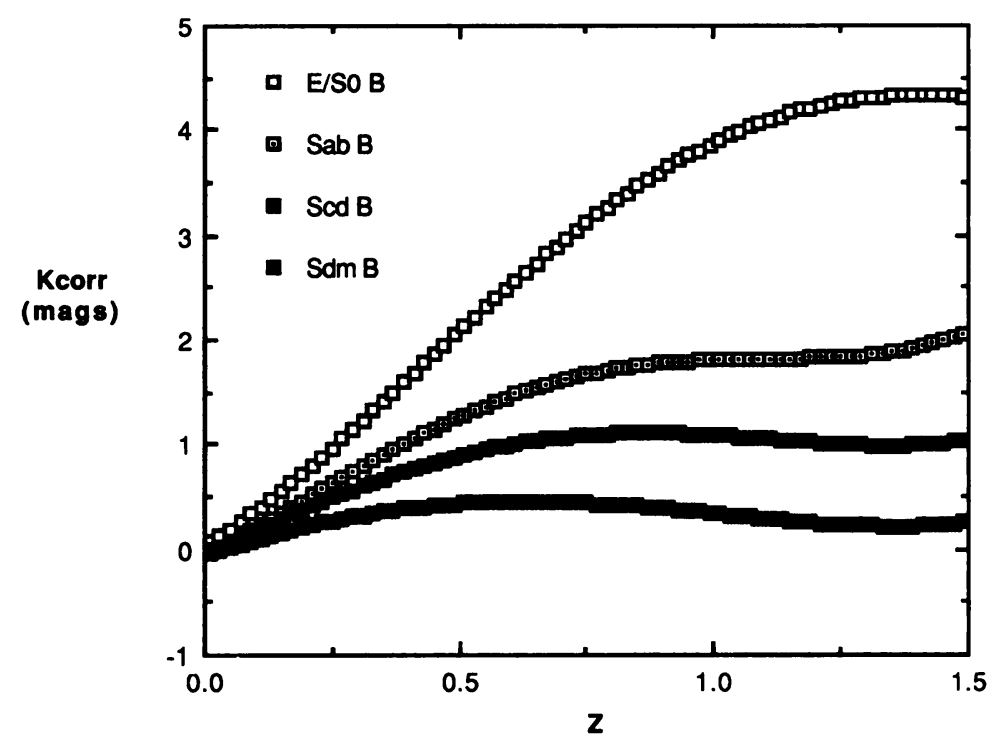

Figure 1. The B K-corrections of King and Ellis (1985) for galaxies of different morphological types.

Observations of galaxies from the OAO-2, ANS and IUE satellites have been used to derive K-corrections, and these observations have been summarised by Ellis (1982). King and Ellis (1985) compared the IUE spectra of elliptical galaxies (Bruzual and Spinrad, 1981 and Ellis, unpublished), with 18 broad-band UV measurements from OAO-2 and ANS. They also compared the UV spectra of later types inferred from the ANS observations of Coleman et al. (1980) with the average of 40 broadband UV observations from the OAO-2 satellite and 70 from the ANS satellite. They found reasonable agreement between the different observations for all morphological types to wavelengths as short as $1500 \AA$. They concluded that the B Kcorrections should be reliable to $z=1.5$ and the R K-corrections to $z=3.0$. The B K-corrections adopted by King and Ellis are shown in Figure 1. The results show that the $\mathbf{K}$ dimming for early types is as much as $4^{m}$ at $z=1$ but much less for the later types. Further improvements 
in our knowledge of the K-corrections now await new observations of local galaxies from the Hubble Space Telescope.

\subsection{The Galaxy Luminosity Function}

Although the basic form of the galaxy luminosity function has been known for some time (see review by Felten (1977), also Efstathiou et al. (1989), until recently very little has been known about the galaxy luminosity function's dependence on colour, particularly at faint magnitudes. Using the B-V colours of Metcalfe et al. (1989a) for $350 \mathrm{~B} \leq 17^{\mathrm{m}}$ galaxies in the redshift survey of Peterson et al. (1986), and the colours and redshifts from the survey of Kirshner et al. (1978), Shanks et al. (1989) have derived the luminosity functions of three (rest) colour classes. The results are shown in Figure 2. A nonparametric method due to Peebles (private communication) was used to calculate the luminosity functions and the maximum likelihood technique of Sandage et al. (1979) was used to fit the Schechter luminosity function parameters, $M^{*}$ and $\alpha$. As can be seen in Figure 2, the reddest galaxies, if anything, seem to show a
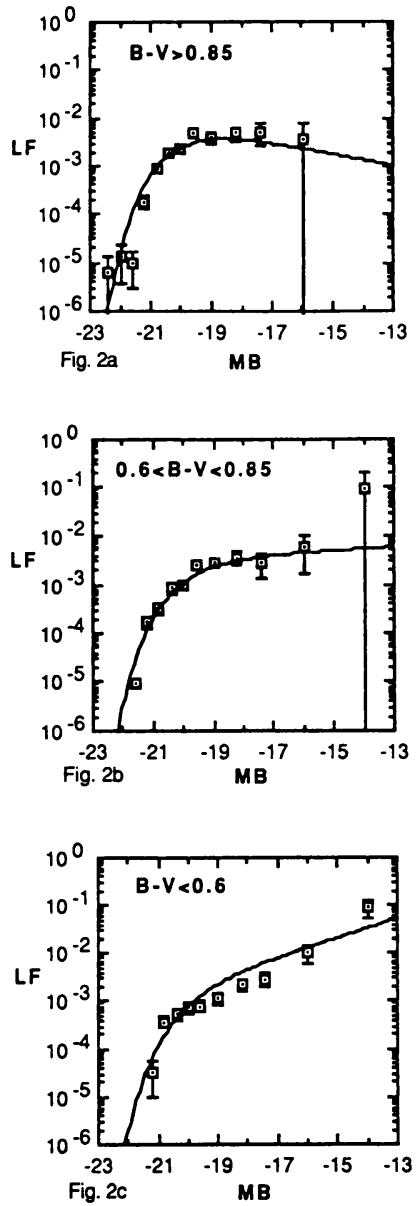
turnover in their luminosity function at faint magnitudes $(\alpha=-0.7)$, whereas the luminosity function of the bluest galaxies seems to rise steeply toward fainter magnitudes $(\alpha=-1.5)$. The solid lines in Figure 2 show the maximum likelihood Schechter function fits for the three different classes. The difference between the luminosity functions of the reddest and bluest galaxies is reasonably significant, with the $2 \sigma$ contours of the maximum likelihood fits nonoverlapping.

Support for this result comes from the data of Mobasher et al. (1986), who give B-H colours and $\mathbf{M}_{H}$ magnitudes for a subset of 150 of the above galaxies. These authors plotted B-H vs. $\mathbf{M}_{H}$ and found a strong colour-magnitude relation despite the fact that their sample was spiral dominated (see their Figure 5). With their lack of faint red galaxies and excess of faint blue galaxies, we take this result to be in strong support of our own. Mobasher et al. interpreted their result in terms of a physical colour magnitude relation; whatever the cause, the effects on the luminosity function are clear.

In terms of $n(m)$ modelling, it is tantalising that the luminosity function of the blue galaxies is nearly, but not quite, steep enough to dominate the counts; this would require $\alpha=-2$ rather than $\alpha=-1.5$. However, we shall see later that the high numbers of faint galaxies detected have some implications for the interpretation of the counts at faint limits.

Figure 2. (a) Red galaxy luminosity function. The solid line represents the maximum likelihood Schechter function fit with $\mathrm{M}^{*}=-19.6\left(H_{o}=100 \mathrm{~km} \mathrm{~s}^{-1} \mathrm{Mpc}^{-1}\right), \alpha=-0.7$. (b) Intermediate galaxy luminosity function. The fitted Schechter function parameters are $M^{*}=-19.9, \alpha=-1.1$. (c) The blue galaxy luminosity function. The fitted Schechter function parameters are $M^{*}=-20.0$, $\alpha=-1.5$. 


\subsection{Count Normalization}

As with any number count it is always important to carefully check the normalization of the count at bright magnitudes. An accurate count normalization allows, for example, exact estimation of where evolution begins to dominate $n(m)$. In Figure 3, I show the galaxy $n(B)$ relation in the range $11^{m} \leq B \leq 25^{m}$. At bright magnitude limits, $B \leq 20 .^{m} 5$, the data is taken from the photographic counts of Shanks et al. $(1984,1989)$. These counts mainly apply to the 15 randomly selected UKST fields $\left(\approx 220 \mathrm{deg}^{2}\right)$ used for the Durham redshift surveys. The COSMOS measuring machine magnitudes have been calibrated by CCD photometry. For $21^{m} \leq \mathrm{B} \leq 25^{m}$ photometry is taken from Metcalfe et al. (1989b) and is based on deep CCD photometry of 12 fields covering $250 \mathrm{deg}^{2}$. A no-evolution model is also shown in Figure 3 (slightly in advance of the natural progression in this review). It was normalized at $B=18 .^{m} 5$ and it can be seen that in the range $18^{m} \leq B \leq 22^{m}$ the no-evolution model fits very well. At fainter magnitudes the model underestimates the counts; this may be expected due to evolutionary effects at high redshift. Less expected is the poor fit at $B \leq 17^{m}$, where the model now seriously overestimates the counts by a factor of about 2 .

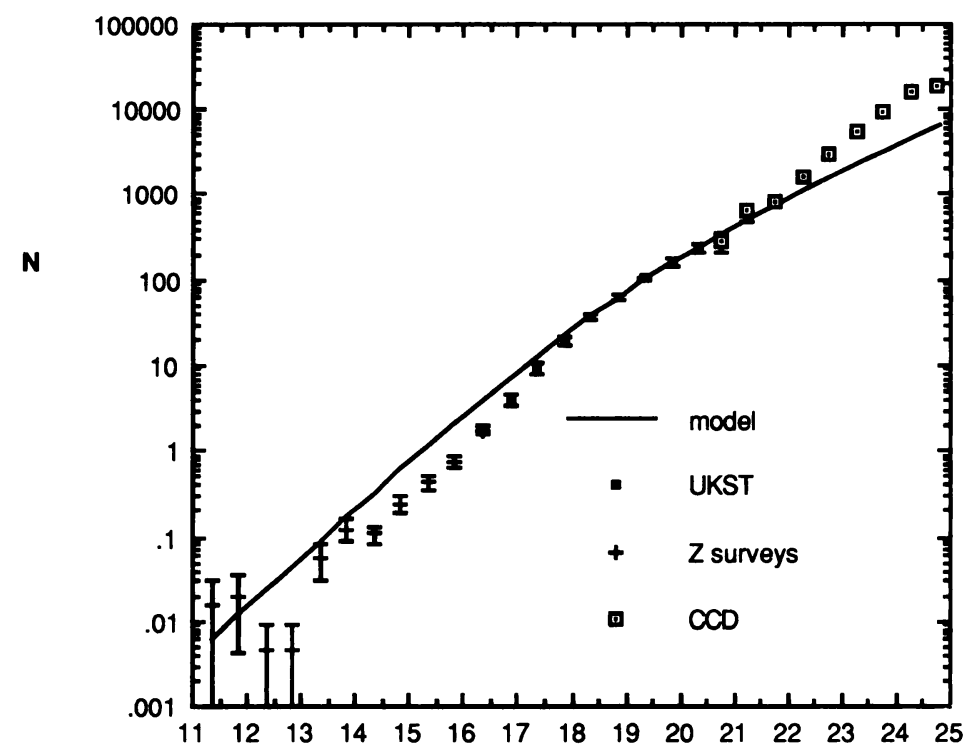

B

Figure 3. The differential, galaxy $n(B)$ relation derived self-consistently from Durham data. At bright magnitudes the data comes from CCD calibrated COSMOS machine measurements of UK Schmidt plates; at faint magnitudes the counts are from the CCD observations of Metcalfe et al. (1989b).

In trying to explain the poor fit at bright magnitudes, it might first be suspected that it could be due to a technical problem; photographic saturation can cause luminosities of bright objects to be underestimated. However, the available CCD photometry suggests that at least in the range $B \geq 14^{m}$ these problems are not too serious (Metcalfe et al. 1989c). Next, the deficiency might be thought to be due to a statistical fluctuation. However, the field-to-field error bars shown in Figure 3 suggest that it is a very significant effect. Also using new direct 
transforms to Zwicky magnitudes, Shanks et al. (1989) find that the Zwicky counts show good agreement for $B \leq 14^{m}$ and these of course include contributions from the Virgo and Coma clusters. The third explanation is that it is due to evolution. However, the problem here is that we already have luminosity functions for galaxies at $B=21 .^{m} 5$ (Broadhurst et al. 1988; Shanks et al. 1989) and these show little evidence for evolution. The final possibility is that the deficiency is due to galaxy clustering; this would imply that the local region is underdense in galaxy numbers to order of $50 \%$. The only problem with this explanation is that the "hole" in the galaxy distribution would have to be uncomfortably large $\left(\approx 150 h^{-1} \mathrm{Mpc}\right)$. However, the number-redshift relation in the $B=17^{m}$ surveys may also give some supporting evidence to this "hole" hypothesis.

For our purposes here we are going to assume that the $B \leq 17^{m}$ galaxy counts are affected by galaxy clustering inhomogeneities and normalize in the range $18^{m} \leq \mathrm{B} \leq 22^{m}$. This approach has the virtue of being conservative, at least in the sense of reducing the amount of evolution that would otherwise be required at $B \geq 22^{m}$.

\subsection{Deceleration Parameter}

The final ingredient needed for a no-evolution $\mathrm{n}(\mathrm{m})$ model is the cosmological deceleration parameter, $\mathrm{q}_{o}$. At bright and intermediate magnitudes the $\mathrm{q}_{o}$ dependence is low since its effect on the luminosity distance and the volume element tend to cancel. However, there have been suggestions that the effects of $\mathrm{q}_{o}$ at very faint limits may be substantial and this possibility will be considered in detail in Section 5 .

\subsection{Comparison of No-Evolution Models and Data}

In Figures $4 a$ and $b$ we show the B and R counts of Metcalfe et al. (1989b) and Tyson (1988) (only in the $\mathrm{B}$ band) compared with the no-evolution model predictions using the parameters described above. To the limits shown here $\left(B=25^{m}, R=23 .^{m} 5\right)$, the data is still not confusion limited and the predicted galaxy redshifts in non-evolving models are still within the range of validity of the $\mathrm{K}$-corrections. As we have already seen, the counts at $\mathrm{B}=25^{m}$ are 3 times higher than predicted by the models. Thus by inputting the most recent parameters into the non-evolving model and comparing it with the most recent CCD data, the original conclusion of Kron (1979) is confirmed. Figure $4 b$ shows that evolution is also required to fit the $R$ counts; here at $\mathrm{R}=23^{m}$ the models underestimate the counts by a factor of 2 . Figure 5 compares the $n(B-R)$ distribution for $24^{m} \leq B \leq 24 .^{m} 5$ to the no-evolution model. Again an excess of blue galaxies is seen at $\mathrm{B}-\mathrm{R} \approx 1 .^{m} 1$, although for redder galaxies the no-evolution model seems to fit quite well. The galaxies in the peak at $\mathrm{B}-\mathrm{R} \approx 1 \cdot^{m} 1$ are too blue to be unevolved galaxies of any morphology with $z \leq 0.8$.

\section{EVOLUTIONARY MODELS}

Since the no-evolution models do not fit the data, we next consider evolutionary models for the different types. For early types the simplest evolutionary models are the c-models which simply allow the stellar Hertzsprung-Russell diagrams to evolve after an initial burst of star formation. These models were considered by Tinsley and Gunn (1976) who showed that typically the amount of evolution produced is about $-1^{m}$ in the rest $V$ band at $z=1$. Tinsley (1978) and Bruzual (1981) also considered models where the star formation rate decreased exponentially with time. These so-called $\mu$-models look very similar to c-models at late times for $\mu \geq 0.5$ 

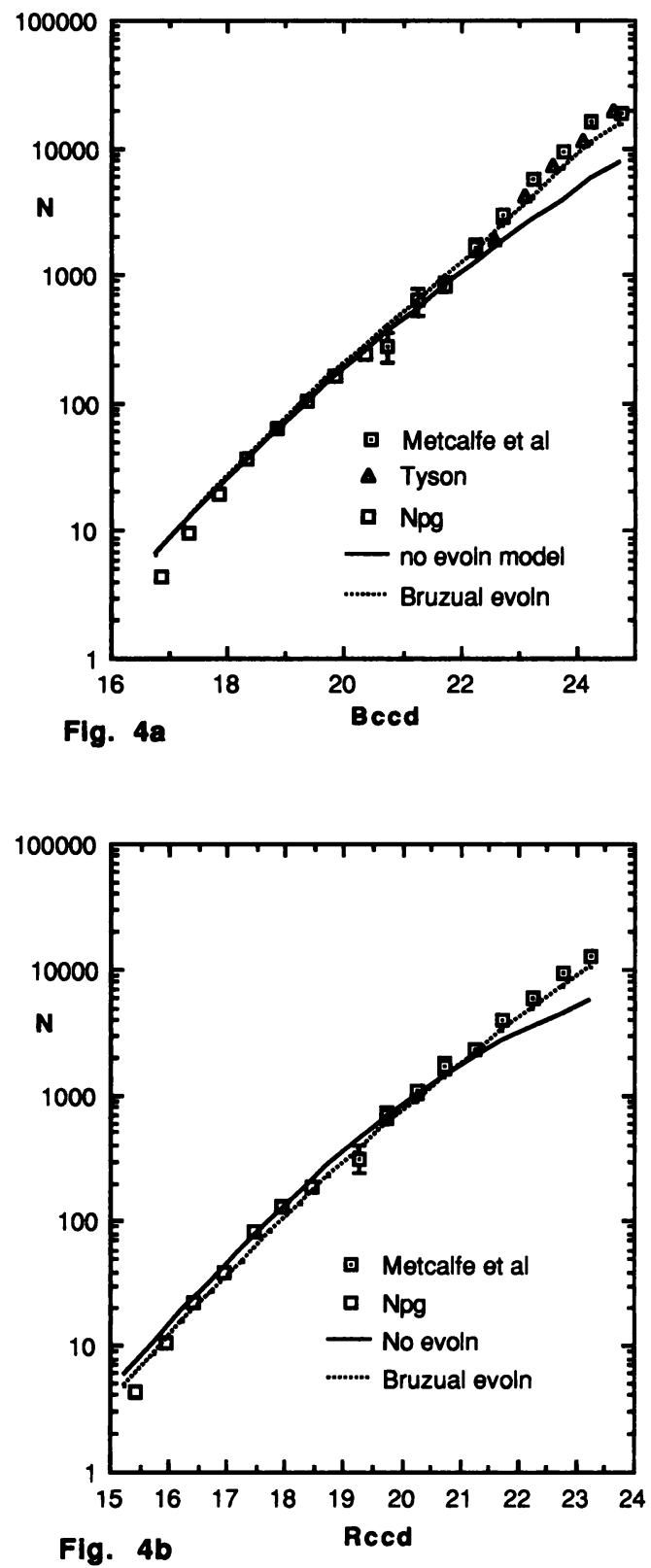

Figure 4. (a) The differential B number counts (per $\operatorname{deg}^{2}$ per $0 .{ }^{m} 5$ ) compared with the non-evolving model and to an evolutionary model based on the results of Bruzual (1981). The evolutionary models assume $\mathrm{q}_{o}=0.01$. (b) As $(a)$ for the $\mathrm{R}$ number counts. 


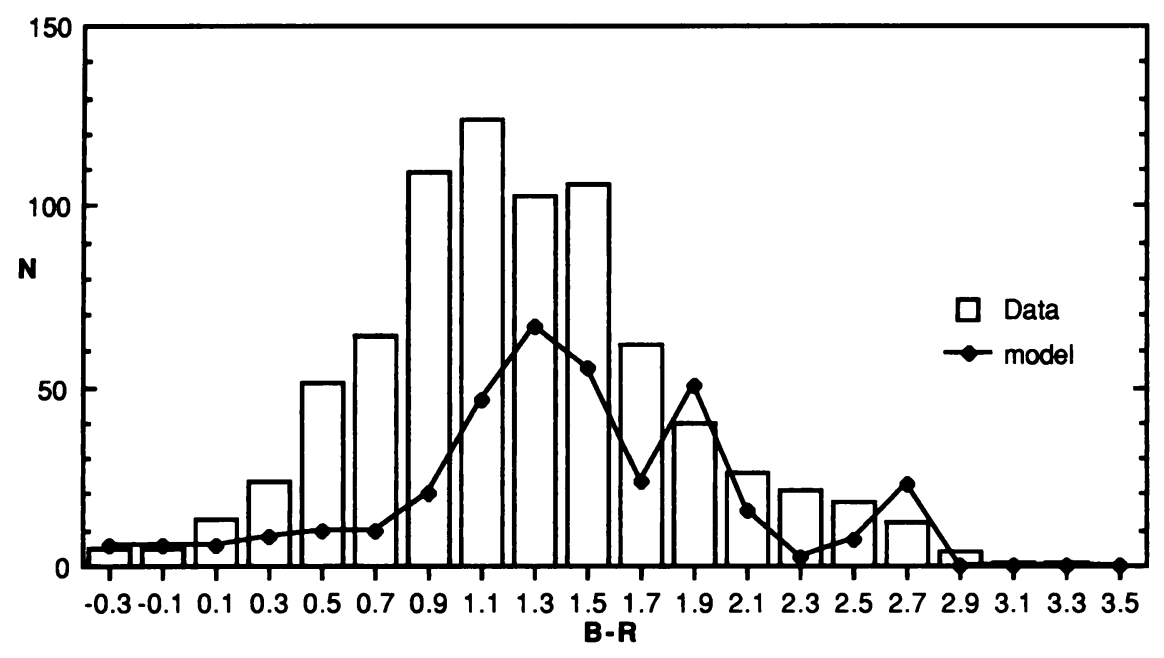

Figure 5. The $n(B-R)$ distribution of Metcalfe et al. (1989b) in the range $24^{m}<B<24 .^{m} 5$, compared with the no-evolution model.

(i.e., for exponential decay times of the star formation rate shorter than $1.5 \mathrm{Gyr}$ ). The conclusion was that the colours of present day early-type galaxies were best fitted by models which assumed 16 Gyr ages rather than 9 Gyr ages, given also the assumption of a Saltpeter initial mass function. Guiderdoni and Rocca-Volmerange (1987) have more recently confirmed and extended these conclusions.

For later types, Tinsley (1978) suggested that spirals are best modelled with a constant star-formation rate; a dominant initial burst of star formation here results in colours which are too red for present day spirals. This means that, generally, spirals only undergo very slow luminosity and colour evolution. Bruzual (1981) again confirmed this conclusion with more detailed models.

Inputting into the $n(m)$ models the most extreme $\mu=0.5$ early-type evolutionary model considered by Bruzual (1981), for all early-type galaxies earlier than Sbc and adopting his evolutionary models for Scd's and Sdm's produces the prediction shown by the dashed line in Figure 4. This model produces a better fit than the non-evolving model but still slightly underestimates the $B$ count at $B=25^{m}$.

Despite its improved fit, the parameters of the evolutionary model are still very arbitrary in terms of the assumed Initial Mass Functions, galaxy ages etc. Unfortunately current cosmological theories do not usefully constrain such parameters; there is no real consensus yet as to how or when galaxies formed. For example, Partridge and Peebles (1967) suggested that young galaxies might form at $z=20$ in a rapid collapse leading to a bright initial burst of star formation. Galaxies would be expected to form in this way, for example, in the baryon isocurvature model of Peebles (1987). In this picture, therefore, young galaxies might be expected to be most easily detected as luminous objects in the near-infrared. However, other theories of galaxy formation such as biased cold dark matter (Frenk et al. 1988) suggest that galaxy formation might take place as late as $z=1$. Here galaxies would form slowly with perhaps some star formation taking place before the galaxy subcomponents finally merged together. This leaves young galaxies appearing relatively blue, extended and with low surface brightness (see 
Baron and White 1987). With the wide range of possible evolution histories allowed between, and even within, the various galaxy formation models, there seems to be little prospect of quick progress in understanding galaxy counts from purely theoretical inferences about galaxy evolution.

\section{REDSHIFT INFORMATION}

If the redshift distribution of faint galaxies were known then this would strongly constrain models of galaxy luminosity evolution. The effort to obtain redshifts has led to two somewhat different interpretations of the $n(z)$ data.

On the one hand, Broadhurst et al. (1988) found that the $\mathrm{n}(z)$ from their $\mathrm{B}=21 .^{m} 5$ limited galaxy redshift survey was well fitted by a no-evolution model, with no sign of any high redshift tail as required if strong evolution by intrinsically bright galaxies were the explanation of the steep $n(m)$ slope at faint magnitudes. No-evolution models have also been found to fit the slightly deeper redshift surveys of Koo and Kron (1988) and Colless et al. (1989). Broadhurst et al. (1988) therefore suggested that intrinsically faint galaxies might evolve the fastest; the $\mathrm{n}(\mathrm{m})$ slope would then be steepened by galaxies which were still at relatively low redshifts. This would imply that the galaxy luminosity function steepens with redshift; it would also imply that the evolved $B \approx 24^{m}$ galaxies may only be at redshifts of order unity.

Cowie and Lilly (1989), however, firstly argued that, irrespective of their redshift, the blue galaxies at $\mathrm{B}=24^{m}$ could form a large proportion of the metals in the present Universe. This argument is simply based on the fact that these blue galaxies are either plentiful, low luminosity galaxies or scarce, high luminosity galaxies. Either way, simple assumptions suggest that their blue star populations will produce large amounts of metals. Secondly, they obtained a spectrum of a single $B \approx 23^{m}$ galaxy which showed Lyman $\alpha$ emission line at $z=3.38$. They confirmed that it was Lyman $\alpha$ through detection of a Lyman limit via $U$ band CCD photometry. On these grounds they suggested that many of the blue, metal-forming galaxies have $z \approx 3$ and that they were perhaps seeing a large population of galaxies undergoing an initial burst of star formation.

The debate as to the redshift of the blue galaxies is finely balanced at present. Against the observation of the $z=3.38$ galaxy it can be said that some evolution in $n(B-R)$ can be seen as bright as $B=22 .^{m} 5$ and here the redshift survey of Colless et al. (1989), for example, already suggests that these are not galaxies at $z \approx 3$. Also, the high fraction of $B=24^{m}$ galaxies that are detected in the U passband by Majewski (1989) suggests that if the evolved galaxies have strong Lyman limits then their redshifts must be smaller than $z=3$. On the other hand there is still no positive evidence for steepening of the galaxy luminosity function with redshift. The luminosity functions derived from the survey of Broadhurst et al. (1988) by Shanks et al. (1989) look very similar to the those seen in Figure 2. Also Guiderdoni and RoccaVolmerange (1989) have claimed that the assumption of their $\mu=0.5$ models for intrinsically bright early types may give reasonable fits to both the $n(m)$ and $n(z)$ relations without any call for faster evolution for faint galaxies. Further observations are clearly required to resolve the question of the redshift of $24^{\mathrm{m}}$ galaxies and some new results on this topic have been described by Tyson (1989, this volume).

\section{IMPLICATIONS FOR $\mathrm{q}_{o}$}

As indicated already, the effects of $q_{o}$ on $n(m)$ are small at intermediate magnitudes where generally the effects of evolution dominate. However, Koo (1989) has suggested that the sheer 
numbers of galaxies detected in Tyson's surveys might argue against $\mathrm{q}_{o}=0.5$ models. The argument is based on the assumption that galaxies have strong Lyman limit systems. If so then this suggests that galaxies will only be detected to $z=4$ in the $B$ band; this would then mean that $\mathrm{q}_{o}=0.5$ models would have four times less volume available to them as $\mathrm{q}_{o}=0.05$ models. Koo argued on the basis of a local density of 0.0015 "observable" galaxies per $\mathrm{Mpc}^{3}\left(H_{o}=50\right)$ that Tyson was already observing two times more galaxies at $\mathrm{B}=27^{m}$ than predicted for the $\mathrm{q}_{o}=0.5$ case.

Guiderdoni and Rocca-Volmerange (1989) have made detailed models on a variant of this idea. Instead of the Lyman-limit producing a redshift cut-off, they argued that if the redshift of galaxy formation were low then low values of $\mathrm{q}_{o}$ might be preferred by Tyson's counts if galaxies evolved at the same rate irrespective of their intrinsic luminosity, i.e., assuming a pure-luminosity evolution model.

Clearly, the large numbers of faint galaxies detected at faint limits in our local galaxy luminosity function ensure that Koo's argument is not independent of the model of galaxy luminosity evolution. Thus if the form of the evolution suggested by Broadhurst et al. applies then the $\mathrm{q}_{o}=0.5$ model may then be rescued by the fact that their rapid evolution allows the detection of more intrinsically faint galaxies at high redshift. In the case of Guiderdoni and Rocca-Volmerange, the adoption of the Broadhurst et al. model would mean the dropping of their assumption of pure luminosity evolution and their argument against high values of $q_{o}$ would then fail.

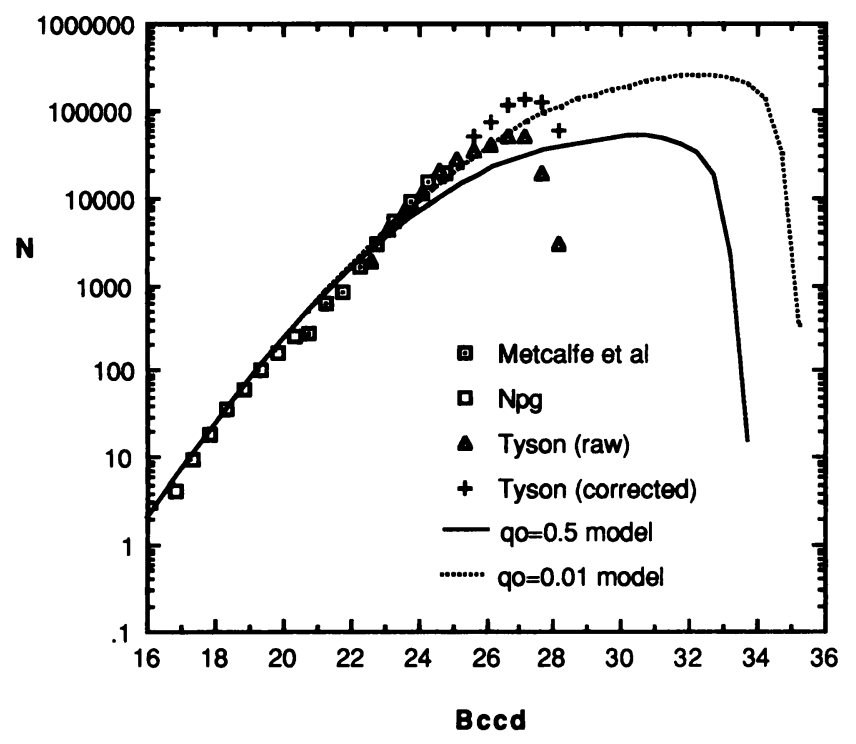

Figure 6. The B count data compared with simplified models in which K-corrections are set to zero. The model counts are cut off at $z=4$. The fit to the form of the counts could be improved for both models by inclusion of ad hoc amounts of luminosity evolution. The models show that if Tyson's $n(B)$ turnover is real, the integrated galaxy count is too high for the low $q_{o}$ model compared to either Tyson's raw or corrected data.

In fact it can be argued that the form of Tyson's B counts actually supports the $\mathrm{q}_{o}=0.5$ model. In Figure 6 are shown two simple models that respectively assume $q_{o}=0.01$ and 0.5 . 
Both models have redshift cut-offs at $z=4$ corresponding to Koo's assumption that galaxies at $z>4$ may not be seen due to the presence of the Lyman break in the B band. To simplify matters the $\mathrm{K}$-corrections were set to zero to roughly simulate the effects of evolution. As can be seen, both models produce turnovers in the counts: the $q_{o}=0.01$ model at $B=34^{m}$ and the $\mathrm{q}_{o}=0.5$ model at $\mathrm{B}=32^{m}$. Although the resulting fits to Tyson's counts are poor, we emphasise that this could be improved in the case of either model by adding ad-hoc evolutionary corrections. The important point is that the number of galaxies that are in principle visible is $2.8 \times 10^{6} \mathrm{deg}^{-2}$ for the $\mathrm{q}_{o}=0.01$ case and $5.9 \times 10^{5} \mathrm{deg}^{-2}$ for the $\mathrm{q}_{o}=0.5$ case, integrating the $\mathrm{n}(\mathrm{m})$ count to $\mathrm{B}=40^{m}$ in each case. This compares to $6.3 \times 10^{5} \mathrm{deg}^{-2}$ from integrating Tyson's corrected B count past his turnover. I would argue that the larger number of galaxies seen in the $\mathrm{q}_{o}=0.01$ case cannot be easily explained away if, as Tyson has claimed at this meeting, the turnover seen in his counts at $B=27^{m}$ is real and he has detected in the B band all the galaxies with $z<4$. Thus if Tyson's $\mathrm{n}(\mathrm{B})$ turnover is real, then Koo's argument is reversed, at least using the luminosity functions adopted here, and too few galaxies are seen to be compatible with a simple, low $\mathrm{q}_{o}$ model.

In this case mergers (i.e., more galaxies at high redshift) only make the problem worse for low $\mathrm{q}_{o}$ models. Clearly, however, one way out for low $\mathrm{q}_{o}$ models is if galaxies were to form at $z<4$. When $z_{\text {formation }}$ was reduced to $z=1.8$ in the above, low $\mathrm{q}_{o}$ model, only then did the numbers of galaxies produced match the data. On this basis, models that assume low $\mathrm{q}_{o}$ and high $z_{\text {formation }}$ may be discriminated by the turnover in Tyson's B counts; this could have serious consequences for the baryon isocurvature model, for example. However, this argument totally depends on the turnover in Tyson's $n(B)$ being real. If the counts actually continue to rise steeply then Koo's original conclusion would apply, with low $\mathrm{q}_{o}$ high $z_{\text {formation }}$ models being preferred. Obviously it is important for other observers to try and confirm the reality of the $n(B)$ turnover claimed by Tyson.

\section{IMPLICATIONS FOR THE EBL}

In the $B$ band, the fact that the galaxies with $22^{m} \leq B \leq 27^{m}$ account for $10 \%$ of the EBL upper limit means that the $B$ counts could extend with their $d(\log N) / d B \approx 0.5$ slope to $B \approx 32^{m}$. However, if the EBL limits were lower by a factor of $\approx 5$ then a flattening of the $n(B)$ relation would be implied. Such a turnover is required by low $z_{\text {formation }}$ and by high $\mathrm{q}_{o}$ models. Conversely, if the turnover seen at $\mathrm{B}=27^{m}$ by Tyson is real, then $90 \%$ of the current optical EBL upper limits is not accounted for by galaxies.

In the far-UV it should be pointed out that, despite the trend in the optical for steeper count slopes at bluer passbands, very little EBL will be contributed by $\mathrm{B}>24^{m}$ galaxies. This argument is based on the likelihood that many galaxies will show very little flux below the 912 $\AA$ Lyman limit; either the galaxies will contain neutral gas which will absorb the light from young stars or there will be no neutral gas and hence no young blue stars. Since on any reasonable model the galaxies at $\mathrm{B}>24^{m}$ will have $z>1$, these galaxies will contribute little UV EBL and the EBL at $2000 \AA$ may therefore be best predicted by further investigation of the UV spectra of galaxies with $z \leq 1$. The sensitivity of the UV EBL to cosmology is likely to be low, since the cosmological volume differences at $z=1$ are small.

There are several indications that galaxies may contribute a significant IR background. Firstly, there is the possibility that the Partridge and Peebles (1967) model is correct and that bright, primeval galaxies will be seen at $z=20$. Secondly, there is the possibility that even young galaxies at low redshift may re-radiate much of their light in the IR if they contain large quantities of dust. The IR starburst population of Rieke et al. (1985) gives clear evidence that 
such a population may exist locally. Finally, Rowan-Robinson and Carr (1988) have suggested that the steep IRAS galaxy counts may imply strong evolution for these galaxies and hence a potentially large IR EBL.

Despite these arguments, the implications for the IR EBL from the optical galaxy count results are still unclear. Firstly, the optical $\mathrm{n}(\mathrm{m})$ data show too many blue galaxies at $\mathrm{B}=25^{\mathrm{m}}$ rather than too few. Thus the data do not demand either $z=20$, very red primeval galaxies or dust re-radiation in young galaxies at lower redshifts; of course, neither do the data rule out these possibilities. Secondly, if Tyson's $n(B)$ turnover is to be taken seriously then the argument in Section 5 suggests that high redshifts of galaxy formation are unlikely, at least for low values of $q_{o}$. Finally, if the "hole" interpretation of the steep galaxy $n(m)$ at $B \leq 17^{m}$ is correct and if the same explanation also applies to the IRAS $n(m)$, then the amount of evolution implied would be reduced, reducing in turn the possible contribution of IRAS galaxies to the IR background. In conclusion, at present there are reasonable arguments both for and against a large contribution from faint galaxies to the infrared backgrounds.

\section{CONCLUSIONS}

We have reviewed the parameters of number count models including the K-corrections, galaxy luminosity functions, the count normalization and the evolutionary models. In particular, we have discussed the new evidence that the luminosity function of blue galaxies is different from that of red galaxies. The results of Shanks et al. (1989) suggest that the blue galaxy luminosity function rises steeply toward faint magnitudes whereas the red galaxy luminosity function is flat or even drops toward fainter magnitudes. We have also discussed the uncertainty in the count normalization at bright magnitudes. The local galaxy density, as measured in the Durham B<17 mag redshift survey fields, appears underdense by a factor of 2 compared with fainter magnitudes. We have suggested that this might be due to a galaxy clustering inhomogeneity on $150 \mathrm{~h}^{-1} \mathrm{Mpc}$ scales but other possibilities have also been discussed. Whatever the reason for this effect, we have chosen to normalize our count models at fainter magnitudes $\left(B=18 .^{m} 5\right)$. Comparing the new, non-evolving models with the most recent count data by Metcalfe et al. $(1987,1989 \mathrm{~b})$ and Tyson (1988), we find that the non-evolving models underestimate the counts by a factor of 3 at $B=25^{m}$ and a factor of 2 at $R=23 .^{m} 5$, confirming the results of previous, similar analyses. The detected evolution takes the form of excess numbers of blue galaxies with $\mathrm{B}-\mathrm{R}=1 .^{m} 1$ for magnitudes $\mathrm{B}>22 .^{m} 5$.

We have briefly reviewed theoretical models for galaxy evolution and find that the input parameters, from the initial mass function for stars to the underlying galaxy formation model, are still not well enough specified to make possible unambiguous predictions for $n(m)$. More observational constraints are required; one great uncertainty is the redshift distribution of the galaxies for $B>23^{m}$. The debate between Cowie and Lilly (1989), who have argued that $B=23^{m}$ galaxies may lie at $z \approx 3$, and Broadhurst et al. (1988), who have suggested that such galaxies may only lie at $z \approx 1$, has been discussed. The conclusion is that at present these arguments are finely balanced and more information in terms of $B>23^{m}$ redshift surveys is required to settle this issue.

The suggestions by Koo (1989) and by Guiderdoni and Rocca-Volmerange (1989) that the large numbers of galaxies already detected by Tyson at $B=27^{m}$ may argue for a low $\mathrm{q}_{o}$ model have been considered. However, the large numbers of faint blue galaxies detected in our local galaxy luminosity function weakens this conclusion, especially if intrinsically faint galaxies evolve faster than bright galaxies in the manner suggested by Broadhurst et al. (1988). Indeed, it has been argued here that if the turnover claimed by Tyson in the $n(B)$ counts is 
real, then high $\mathrm{q}_{o}$ (or low $z_{\text {formation }}$ ) models may actually be preferred. It is therefore important for new efforts to be made to establish better the form of the $B$ galaxy counts at $B=27^{m}$.

Finally, we have reviewed the implications of the $n(m)$ models for the extragalactic background light. In the infrared, the galaxy count data make reasonably weak inferences for the faint galaxy contribution to the EBL. Although many evolved blue galaxies are seen in deep optical number counts, this does not rule out the possibility of a significant contribution to the infrared EBL from, for example, galaxy starlight being re-radiated by dust into the infrared. However, in the optical bands, if the turnover claimed in Tyson's $n(B)$ relation is real, the stronger conclusion is that faint galaxies may not account for more than $10 \%$ of the current EBL upper limit. In the UV we have suggested that if galaxies show a strong Lyman limit then the contribution to the far-UV EBL by galaxies with $B>24^{m}$ (and thus $z>1$ ) may also be very low.

Acknowledgments - I thank N. Metcalfe, R. Fong, D. Hale-Sutton and L.R. Jones for allowing me to use the results on the galaxy luminosity function and its normalization in advance of publication. I thank the Royal Society for its continued support of my research.

\section{REFERENCES}

Baron, E., and White, S. D. M. 1987, Astrophys. J., 322, 585.

Boyle, B. J., Shanks, T. and Peterson, B. A. 1988, Mon. Not. R. Astr. Soc., 235, 935.

Broadhurst, T. J., Ellis, R. S., and Shanks, T. 1988, Mon. Not. R. Astr. Soc., $235,827$.

Bruzual, G. 1981, Ph.D. thesis, Univ. of California at Berkeley.

Bruzual, G., and Spinrad, H. 1981, in The Universe at Ultraviolet Wavelengths ed. Chapman, R. D. Dordrecht:Reidel.

Coleman, G. D., Wu, C.-C. and Weedman, D. W. 1980, Astrophys. J. Suppl., 43, 393.

Colless, M. C., Ellis, R. S. and Taylor, K. 1989, in The Epoch of Galaxy Formation, eds. Frenk, C. S. et al., Dordrecht: Kluwer, 359.

Cowie, L. L. 1988, in The Post Recombination Universe, eds. Kaiser, N., and Lasenby, A. N., Dordrecht: Kluwer, 1.

Cowie, L. L., and Lilly, S. J. 1989, Astrophys. J., 336, L41.

Dube, R. R., Wickes, W. C. and Wilkinson, D. T. 1977, Astrophys. J., 215, L51.

Efstathiou, G. P., Ellis, R. S. and Peterson, B. A. 1989, Mon. Not. R. Astr. Soc., 232, 431.

Ellis, R. S. 1982, in The Origin and Evolution of Galaxies, eds. Jones, B. J. T., and Jones, J., Dordecht: Reidel, 255.

Felten, J. E. 1977, Astr. J., 82, 861.

Frenk, C. S., White, S. D. M., Davis, M., and Efstathiou, G. 1988, Astrophys. J., 327, 507.

Guiderdoni, B., and Rocca-Volmerange, B. 1987, Astron. Astrophys., 186, 1.

Guiderdoni, B., and Rocca-Volmerange, B. 1989, Astron. Astrophys., in press.

King, C. R. and Ellis, R. S. 1985, Astrophys. J., 288, 456.

Kirshner, R. P., Oemler, A., and Schechter, P. 1978, Astr. J., 83, 1549.

Koo, D. C. 1989, in The Epoch of Galaxy Formation, eds. Frenk, C.S. et al., Dordrecht: Kluwer, 71.

Koo, D. C., and Kron, R. G. 1988, in Towards Understanding Galaxies at Large Redshift, eds. Kron, R. G., and Renzini, A., 209.

Kron, R. G. 1979, Ph.D. thesis, University of California at Berkeley.

Majewski, S. R. 1989, in The Epoch of Galaxy Formation, eds. Frenk, C. S. et al., Dordrecht: Kluwer, 85.

Metcalfe, N., Fong, R., Jones, L. R., and Shanks, T. 1987, in High Redshift and Primaeval Galaxies, eds. Bergeron, J. et al., Paris: Editions Frontieres, 37.

Metcalfe, N., Shanks, T., and Fong, R. 1989a, in preparation.

Metcalfe, N., Shanks, T., Fong, R., and Jones, L.R. 1989b, in preparation.

Metcalfe, N., Fong, R., Shanks, T., and Kilkenny, D. 1989c, Mon. Not. R. Astr. Soc., 236, 207.

Mobasher, B., Ellis, R. S., and Sharples, R. M. 1986, Mon. Not. R. Astr. Soc., 223, 11.

Paresce, F., and Jakobsen, P. 1980, Nature, 288, 119. 
Partridge, R. B. and Peebles, P. J. E. 1967, Astrophys. J., 148, 377.

Peebles, P. J. E. 1987, Nature, 327, 210.

Peterson, B. A., Ellis, R. S., Efstathiou, G., Shanks, T., Bean, A.J., Fong, R., and Zen-Long, Z. 1986, Mon. Not. R. astr. Soc., 221, 233.

Rieke, G., Cutri, R., Black, J., Kailey, W., McAlary, C., Lebofsky, M., and Elston, R. 1985, Astrophys. J., $290,116$.

Rowan-Robinson, M., and Carr, B. J. 1988, in The Post-Recombination Universe, ed. Kaiser, N., and Lasenby, A.N., Dordrecht: Kluwer, 125.

Tinsley, B. M. 1978, Astrophys. J., 220, 816.

Tinsley, B. M., and Gunn, J. E. 1976, Astrophys. J., 203, 52.

Sandage, A., Tammann, G. A., and Yahil, A. 1979, Astrophys. J., 232, 252.

Shanks, T., Metcalfe, N., Hale-Sutton, D., and Fong, R. 1989, in preparation.

Shanks, T., Stevenson, P. R. F., Fong, R., and MacGillivray, H.T. 1984, in Astronomy with SchmidtType Telescopes, ed. Cappacioli, M. Dordrecht: Reidel, 499.

Tyson, J. A. 1988, Astr. J., 96, 1.

Tyson, J. A. 1989, in Proc. IAU 139, The Galactic and Extragalactic Background Radiation, ed. Bowyer, S., and Leinert, Ch., Dordrecht:Kluwer Academic Publishers.

J. Lequeux: The lack of bright galaxies you mention reminds me of the lack of bright radio sources long known to exist for the famous $\log N / \log S$ problem. For example, there is a preprint by $P$. Shaver and M. Pierre (ESO No. 637) claiming that they see an excess of strong radio sources in the Molonglo catalog along the supergalactic plane, resulting in a normal $\log N / \log S \approx-1.5$ for the region, the anomalous slope of $\approx-1.9$ being seen near the supergalactic poles only. Can you comment?

T. Shanks: Although the slopes are similar in the optical and the radio region, the explanation for the radio sources is more likely to be evolution, since these bright objects probe to large redshifts $(z \approx 1)$. At the $B=18^{m}$ limit in the optical counts, the average galaxy redshift is only $z \approx 0.1$, making evolution possibly a less likely explanation for the steep slope in the optical data.

J. Peebles: Is the luminosity function not well enough known that it can be used to normalize the predicted $d N / d m$, rather than normalizing to $d N / d m$ at $m \approx 18$ ? If not, does your normalization imply a reasonable-looking value for the luminosity function?

T. Shanks: If I were to take the luminosity function from the $B<17^{m}$ galaxy redshift surveys it would be a factor of $\approx 2$ lower than that obtained by normalizing the model at $B=18^{m}$. 